\title{
Kompression nach Sklerotherapie mit flüssigen und aufgeschäumten Agenzien
}

\author{
T. Noppeney; J. Noppeney; B. Cucuruz \\ MVZ Obere Turnstraße, Nürnberg
}

\author{
Schlüsselwörter \\ Kompression, Sklerotherapie, Kompressions- \\ strümpfe, Dauer Kompression
}

\section{Zusammenfassung}

Einleitung: Die Kompressionstherapie gehört zu den basistherapeutischen Maßnahmen der Phlebologie. Aus einer Befragung unter deutschen Phlebologen wissen wir, dass diese in etwa 30 Prozent der Fälle, Patienten nach Sklerotherapie mit Kompressionsstrümpfen versorgen. Methodik: Um die Frage zu beantworten ob Kompressionstherapie nach Sklerotherapie zu besseren Ergebnissen führt, haben wir eine systemische Literaturrecherche in Medline, Pubmed Central und Embase durchgeführt. Dabei konnten wir fünf prospektive randomisierte Studien, eine prospektive Kohortenstudie und Leitlinienempfehlungen zu diesem Thema identifizieren.

Ergebnisse: Die vorliegende Literatur zeigt, dass Kompressionstherapie bis zu 3 Wochen nach Sklerotherapie von Besenreiser und reti-

Korrespondenzadresse

Dr. Thomas und Dr. Jeanette Noppeney

MVZ Obere Turnstraße

Obere Turnstraße 8-10, 90429 Nürnberg

E-Mail: tn@gefaesszentrum-nuernberg.de kulären Varizen zu einer Verbesserung der Ergebnisse, sowie zu einer Verminderung von Pigmentierungen führt. Die Datenlage zur Schaumsklerotherapie größerer Venen ist uneinheitlich, eine prospektiv randomisierte Studie zeigt keinen positiven Effekt der Kompressionstherapie, eine prospektive Kohortenstudie ergibt einen besseren Sklerosierungserfolg in Abhängigkeit von der Compliance der Patienten. Schlussfolgerung: In den Leitlinien wird im Allgemeinen eine Empfehlung zur Kompressionstherapie nach Sklerotherapie abgegeben, wobei keine Empfehlungen zur Dauer der Kompression erfolgen. Aufgrund der eingeschränkten Datenlage sind weitere Studien nötig um den Benefit der Kompression nach Sklerotherapie deutlich zu belegen.

\section{Keywords}

Compression, sclerotherapy, Duration of Compression, Compression stockings

Phlebologie 2018;47: 21-23

https;//doi.org/10.12687/phleb2408-1-2018

Eingereicht: 15. Dezember 2017

Angenommen: 15. Dezember 2017

English version available at: www.phlebologieonline.de

\section{Summary}

Introduction: Compression therapy is one of the basic methods of phlebology. Interviews with German phlebologists showed that about $30 \%$ of the patients are treated with compression stockings after sclerotherapy. Methods: To figure out if sclerotherapy followed by compression therapy leads to better results a systematic literature research in Medline, Pubmed Central and Embase was performed. To this topic five prospective randomized studies were found, one prospective cohort study and guideline recommendations. Results: The literature shows that compression therapy up to 3 weeks after sclerotherapy of telangiectasia leads to improved results and a reduction of pigmentation. The data on foam sclerotherapy of larger veins differs. One prospective randomized study showed no positive effect after compression therapy. One prospective cohort study showed better results using compression therapy after sclerotherapy in reliance of patient compliance. Conclusion: The guidelines recommend compression therapy after sclerotherapy with no recommendation to the duration of the compression therapy. In behalf of the limited data there are more studies necessary to prove the positive effect of compression therapy after sclerotherapy.

\section{Einleitung}

Die Kompressionstherapie gehört zu den basistherapeutischen Maßnahmen in der Phlebologie. Sie ist in der Lage venöse Beschwerden und Schwellungsszustände zu vermindern, Schmerzen zu lindern und hat einen antiinflammatorischen Effekt. In einer Befragung, die unter deutschen Phlebologen durchgeführt wurde, gaben die Teilnehmer an, dass in etwa 30\% der Fälle, Patienten nach Sklerotherapie mit Kompressionsstrumpf ver- sorgt werden (3). Daraus lässt sich ableiten, dass auch unter deutschen Phlebologen keine einheitliche Meinung besteht, ob eine Kompressionsstrumpfversorgung nach Sklerotherapie zu besseren Ergebnissen führt und für den Patienten einen Benefit darstellt.

\section{Methodik}

Um die Frage zu klären ob Kompression nach Sklerotherapie zu besseren Ergebnis- sen führt, haben wir eine systemische Literaturrecherche in Medline, Pubmed Central und Embase durchgeführt.

Dabei konnten wir 5 prospektiv randomisierte Studien identifizieren, die Kompression versus keine Kompression in verschiedenen Protokollen nach Sklerotherapie verglichen haben, eine prospektive Kohortenstudie, eine Befragung und Empfehlungen aus Leitlinien. 


\section{Ergebnisse}

Eine erste prospektiv randomisierte Studie (RCT), in die 40 Patienten eingeschlossen wurden, hat 4 unterschiedliche Gruppen gebildet: Kompression für 3 Tage, eine Woche, 3 Wochen und keine Kompression (9). Nach Sklerotherapie von Besenreisern und retikulären Varizen konnte in den Kompressionsgruppen deutlich bessere Ergebnisse erzielt werden. Es bestand eine klare Korrelation zur Länge der Kompressionstherapie, je länger die Kompression desto besser waren die Ergebnisse.

Ein weiterer Effekt der sich in dieser Studie zeigte, war eine signifikante Reduzierung der Pigmentierungen in den Kompressionsgruppen.

Ein weiterer RCT mit 96 Patienten hat eine Gruppe mit Kompressionsstrümpfen (23-32 mmHg) für 3 Wochen gegen eine Gruppe ohne Kompression nach Sklerotherapie von Besenreisern und retikulären Varizen verglichen (2).

Die Patientenzufriedenheit und Lebensqualität, gemessen am SF 36, war in beiden Gruppen vergleichbar. Das Ergebnis der Sklerotherapie wurde anhand von Fotografien am lateralen Oberschenkel vor und 52 Tage nach Sklerotherapie durch zwei geblindete Reviewer verglichen. In der Kompressionsgruppe zeigten sich signifikant bessere Ergebnisse $(\mathrm{p}=0,026)$ im Vergleich zur Patientengruppe ohne Kompression.

Es liegt ein weiterer RCT mit 29 Patienten vor, bei denen eine Sklerotherapie von Besenreisern und retikuläre Varizen durchgeführt wurden. (4) Alle Patienten wurden an beiden Beinen verödet und erhielten in der ersten Woche an beiden Beinen einen Kompressionsstrumpf mit 30 bis $40 \mathrm{mmHg}$.

Danach wurde ein Bein mit einem Kompressionsstrumpf 20 bis $30 \mathrm{mmHg}$ für 3 Wochen versorgt, das andere Bein erhielt keine Kompression.

Im Ergebnis zeigte sich eine signifikant geringere Pigmentierung und signifikant weniger Hämatome an den Beinen mit einer zusätzlichen Kompressionstherapie für 3 Wochen.

Ein weiterer von uns gefundener RCT verglich Kompressionsstrumpf $15-20 \mathrm{mmHg}(\mathrm{n}=31)$ vs. keine Kompression $(n=29)$ nach Schaumsklerotherapie nach Vena saphena magna (1).
Die Verschlussrate der Vena saphena magna betrug in beiden Gruppen 100\% nach 28 Tagen. Die Verbesserungen der venösen Beschwerdesymptomatik und der Lebensqualität war vergleichbar in beiden Gruppen. Die Rate von unerwünschten Nebenwirkungen war insgesamt sehr niedrig und in beiden Gruppen vergleichbar ( $>0,05)$. Es zeigte sich in dieser Studie kein Vorteil für die Kompressionstherapie.

In einer prospektiven Kohortenstudie wurde untersucht, welche Faktoren Einfluss auf das Ergebniss der Schaumklerotherapie haben (7).

Untersucht wurden Alter, Geschlecht, Compliance mit Kompressionstherapie nach Intervention, vorausgegangene Varizenchirurgie, einzelne oder multiple Stellen der Sklerotherapie, Konzentration des Sklerosierungsmittels, Volumen des Sklerosierungsmittels und der präinterventionelle Beschwerdegrad.

Eingeschlossen in die Studie wurden 126 Patienten. Sklerosiert wurde die Vena saphena magna $(\mathrm{n}=75)$, die Vena saphena parva $(n=13)$ und die Vena saphena accessoria anterior $(n=8)$. Bei den übrigen Patienten wurden andere Venen oder Vena saphena magna und parva gleichzeitig sklerosiert. Die mediane Nachbeobachtungszeit war 3 Monate. Die Nachbeobachtung erfolgte mit Duplex Sonographie. Es zeigte sich ein Verschluss der Vena saphena magna, parva oder accessorischen Vene in $79 \%$. Der einzige Faktor der mit einem erfolgreichem Verschluss der Zielvene assoziiert war, war Compliance mit Kompressionstherapie $(p<0,05)$. Hinsichtlich des häufigeren Auftretens von Pigmentierungen war der einzige Faktor, der diese $\mathrm{Ne}$ benwirkung signifikant beeinflusste, das weibliche Geschlecht $(\mathrm{p}<0,05)$.

Eine weitere randomisierte prospektive Studie hat verglichen, wie sich eine Bandagierung nach Schaumsklerotherapie für 24 Stunden $(n=61)$ oder für 5 Tage $(n=63)$ auf das Ergebnis auswirkt. Die Endpunkte der Studie war der Aberdeen Varicose Vein Severity Score (AVVSS) und Burford Pain Score 6 Wochen nach Sklerotherapie. Es zeigten sich in beiden Gruppen keine signifikanten Unterschiede im Auftreten einer Phlebitis oder Pigmentierung. Ebenfalls bestanden keine signifikanten Unterschiede in der Verbesserung des AVVSS. Insgesamt zeigten sich keine Vorteile für Kompressionsverbände länger als 24 Stunden.
In Frankreich wurde ein Fragebogen an Gefäßmediziner versendet um zu klären, wie und wie lange Kompression nach Sklerotherapie durchgeführt wird.

366 Befragte haben geantwortet, weniger als 1/3 gab an regelmäßig Kompression nach Sklerotherapie anzuwenden. Am häufigsten wurde Kompression nach Sklerotherapie der Vena saphena magna, parva oder der accessorischen Venen angewendet. Wenn Kompression zur Anwendung ,kam dann meist mit Kompressionstrümpfen 15-20 mmHg (5).

Die Dauer der Anwendung reichte von 48 Stunden bis eine Woche nach Sklerotherapie bei 65\% der Befragten ( $n=193 / 299$ ). Die Anwendung von Kompression nach Sklerotherapie steht klar im Gegensatz zu den Empfehlungen der französischen $\mathrm{Ge}$ sundheitsbehörden, die Kompressionsstrümpfe mit 15-20 mmHg oder 20-36 mmHg über eine Zeit von 4-6 Wochen nach Sklerotherapie empfehlen.

In der europäischen Leitlinie (10) wird ebenfalls Kompressionstherapie nach Sklerosierung empfohlen. Es handelt sich um eine I A Empfehlung.

\section{Diskussion}

Wie sich aus unserer Literaturrecherche ergeben hat, ist die Datenlage zur Kompression nach Sklerotherapie sehr dünn.

Aus der Literatur ergeben sich Vorteile für Kompression nach Sklerotherapie von Besenreisern und retikulären Varizen. Allerdings ist die Aussagekraft einiger dieser randomisierten prospektiven Studien eingeschränkt, da nur sehr kleine Kollektive untersucht wurden. Die optimale Dauer der Kompression nach Sklerotherapie von Besenreisern und retikulären Varizen scheint drei Wochen zu betragen.

Die Datenlage zur Kompression nach Schaumsklerotherapie größerer Venen ist sehr uneinheitlich, in der Studie von Hamel-Desnos (1) ergaben sich keine Vorteile für die Kompressionsgruppe, während in einer prospektiven Kohortenstudie (7) die Compliance hinsichtlich des Tragens von Kompressionsstrümpfen das Ergebnis der Schaumverödung größerer Venen positiv beeinflusst hat. 
Die längerfristige Bandagierung der Beine nach Schaumsklerotherapie scheint keinen Vorteil zu ergeben. Das würde sich auch aus der Studie von Protz (6) ableiten lassen, die klar zeigen konnte, dass der gewünschte Kompressionsdruck von 50 bis $60 \mathrm{mmHg}$ nur bei $10 \%$ der Anwender erreicht worden ist und das der Ruhedruck der Bandagierung nach 4maliger Dorsalflexion des Fußes signifikant abgenommen hat.

Insgesamt ist festzustellen, dass uns zur Beantwortung der Frage, ob Kompressionstherapie die Ergebnisse der Sklerotherapie verbessert, keine ausreichenden Daten zur Verfügung stehen. Gleichwohl wird Kompression nach Sklerotherapie, sowohl in Deutschland (3) als auch in Frankreich (8) in unterschiedlichen Ausmaß und unterschiedlicher Länge angewandt.

Für die Zukunft sind hier aussagefähige gut designte Studien zu fordern, die den Benefit von Kompression nach Sklerotherapie eindeutig belegen.

\section{Interessenkonflikt}

Dr. Thomas Noppeney, Beratervertrag mit Firma Medi GmbH, Bayreuth

\section{Ethische Richtlinien}

Für das Manuskript wurden keine Studien an Menschen oder Tieren durchgeführt.

\section{Literatur}

1. Hamel-Desnos CM, Guias BJ Desnos PR, Mesgard A. Foam sclerotherapy of the saphenous veins: randomised controlled trial with or without compression. Eur J Vasc Endovasc Surg 2010;39 (4): 500-507.

2. Kern P, Ramelet AA, Wintschert R, Hayon D. Compression after sclerotherapy for teleangiectasias and reticular leg veins: a randomized controlled trial. J Vas Surg 2007;45: 1212-1215.

3. Mühlberger D, Wallutis K, Wolff EM, Papapostolou G, Reich-Schupke S, Mumme A, Hummel T. Postoperative compression therapy. A survey in Germany. Phlebologie 2017; 46: 292-296.

4. Nootheti PK, Cadag KM, Magpantay A, Goldman MP. Efficacy of graduatet compression stockings for an additional 3 weeks after sclerotherapy treatment of reticular and teleangiectatic leg veins. Dermatol Surg 2009; 35: 53-57.

5. O'Hare Jl, Stephens J, Parking D, Earnshaw JJ. Radomized clinical trial of different bandage regiments after foam sclerotherapy for varicose veins. Br J Surg 2010; 97: 650-656.

6. Protz K, Heyer K, Dörler M, Stücker M, HampelKalthoff C, Augustin M. KompressionstherapieKentnisse und Anwendungspraxis JDDG 2014.

7. Thomasset SC, Butt Z, Liptrot S, Fairbrother BJ, Makhdoomi KR. Ultrasound guided foam sclerotherapy: factors associated with outcomes and complications. Eur j Vas Endovas Surg 2010; 40: 389-392.

8. Tripey V, Monsallier JM, Morello R, Hamel-Desnos C ; French sclerotherapy and compression. Practice patterns .Phlebology 2015; 30: 632-640.

9. Weiss RA, Sadock NS, Goldman MP, Weiss MA. Postsclerotherapy compression: controlled comprarative study of duration of compression and it's effects on clinical outcome. Dermatol Surg 1999; 25: 105-108.

10. Wittens C, Davies AH, Baekgaard N, Broholm R, Cavezzi A, Chastanet S, deWof M, Eggen C, Giannoukas A, Gohel M, Kakkos S, Lawson J, Noppeney T, Onida S, Pttaluga P, Thomis S, Toonder I, Vuylsteke M et al. Management of Chronic Venous Disease: Clinical Practice Guidelines of the Eur J Vas Endovas Surg 2015; 49: 678-737. 